\title{
Sociologia Perennis? Überlegungen zur Problematik prototheoretischer Aussagen in der Soziologie
}

Es soll Personen geben, welche die Lektüre geisteswissenschaftlicher Texte grundsätzlich mit der Bibliographie beginnen, um sich vorab zu vergewissern, ob der Autor die »richtige« Literatur verwendet hat. Wer bei vorliegendem Aufsatz auf diese Weise vorgeht (resp. vorgegangen ist), wird feststellen, dass das Gros der herangezogenen Schriften bereits älteren Datums ist, und aus diesem Umstand womöglich folgern, der Autor habe offenbar nichts essentiell Neues zum Thema zu sagen.

Um es vorweg zu nehmen: Dieser Eindruck ist korrekt. Tatsächlich greifen die hier gemachten Überlegungen lediglich eine bereits etwas ältere Bemerkung von Thomas Luckmann (1980b: 37) auf, in welcher er auf die irrige Verwechslung der "Idee einer deskriptivphänomenologischen Begründung kumulierender empirischer Wissenschaften ... mit der Idee einer ewigen >Ersten Philosophie« hinweist, und versuchen zu klären, worin diese Verwechslung besteht, welche Gefahr sie mit sich bringt und wie Letzterer zuvorzukommen ist. Luckmanns Hinweis bezieht sich auf eine spezifische Form sozialwissenschaftlicher Theoriebildung, und zwar auf den protosoziologischen Ansatz sowie dessen Idee, einen ausgewiesenen Teilbereich der Soziologie aus dem induktiv-empirisch verfahrenden Korpus der Disziplin auszulagern bzw. diesem in begründender Absicht voranzustellen. Dem Missverständnis eben dieses Unterfangens gilt seine Warnung. Die nachstehenden Ausführungen argumentieren, dass vergleichbare Absichten historisch bereits in anderen soziologischen Theorien verfolgt wurden und dass es sich lohnt, den Blick auf solche Ansätze auszuweiten, ${ }^{1}$ lassen sich doch derart die typischen Probleme einer jeden Grundlegungsabsicht - die Klärung des Verhältnisses von prototheoretischen und empirischen Aussagen sowie des epistemologischen Status ersterer ${ }^{2}-$ als allgemeine behandeln sowie vorhandene Lösungsvorschläge miteinander vergleichen. Hier geschieht dies anhand einer Gegenüberstellung der Protosoziologie luckmannscher sowie schützscher Provenienz mit der reinen Soziologie von Ferdinand Tönnies.

Der somit grundlagentheoretisch ausgerichtete Beitrag wendet sich erstens gegen eine fehlgeleitete Auffassung des epistemologischen Status von Prototheorien (Abschnitt IV) und unterbreitet zweitens einen Vorschlag für ein adäquates Verständnis desselben (V). Vorbereitet wird dies durch einen kurzen Problemaufriss (I), dem eine Darstellung des Ansatzes Tönnies' (II) sowie ein Aufweis von dessen Nähe zur Protosoziologie (III) folgen.

1 Infolge dieser Ausweitung verwende ich im Folgenden den Begriff "prototheoretisch", um im Allgemeinen Aussagen zu kennzeichnen, die "vor-empirischer« Natur sind, während der Begriff "protosoziologisch « im Speziellen auf den Theorieansatz Luckmanns hinweist.

2 Folgendes Beispiel vermag das Problem vielleicht zu veranschaulichen: Wolfgang Sofsky kommt in seinen empirischen Untersuchungen über Konzentrationslager zu dem Ergebnis, diese zeichneten sich u. a. durch eine Tilgung der »exzentrischen Positionalität« (Plessner) der Lagerinsassen aus (vgl. Sofsky 1993: 232). Martin Endreß (2004) hält dem entgegen, die »exzentrische Positionalität" könne als anthropologische Grundkonstante des menschlichen Weltbezugs niemals aufgehoben werden, müsse also selbst in Konzentrationslagern in irgendeiner Form ihren Ausdruck finden. Hier zeigen sich widersprechende Auffassungen vom Status prototheoretischer Aussagen, die von grundlegender Relevanz für die praktische Forschung sind, insofern sie deren Blickrichtung lenken. 


\section{Problemstellung}

Die von Luckmanns Lehrer Alfred Schütz betriebene Heranziehung phänomenologischer Betrachtungen für die Soziologie folgte - in der Auseinandersetzung mit dem Ansatz Max Webers - erklärtermaßen der spezifischen Absicht einer (Neu-)Begründung von Sozialtheorie und -forschung:

"Aber so großartig Webers Konzeption dieser 'verstehenden` Soziologie ist, auch sie beruht auf einer Reihe stillschweigend gemachter Voraussetzungen, deren Explikation ein um so dringlicheres Postulat bleibt, als nur eine radikale Analyse der echten und ursprünglichen Elemente des sozialen Handelns eine gesicherte Fundierung der weiteren gesellschaftswissenschaftlichen Arbeit verbürgt« (Schütz 2004: 87).

Die Lösung für die damit angesprochene "Problematik der Sinnsetzungs- und Sinndeutungsphänomene" (93) suchte Schütz in der phänomenologischen Theorie des Bewusstseins. Speziell unter Rekurs auf die Analysen Edmund Husserls wollte er die weitverzweigte Problematik des Begriffs »der sinnhaften und daher verstehbaren Handlung " (87) aufklären. Zu diesem Zweck übernahm Schütz dessen Konzept der Lebenswelt, ${ }^{3}$ deren Analyse nach seinem Tod von Luckmann ausgearbeitet wurde (Schütz \& Luckmann 1979, 1984). Ziel war es, konstitutive - d. h. invariante - Strukturen der subjektiven Weltorientierung herauszuarbeiten, die den gesellschaftlich konstruierten - d. h. variierenden - Wahrnehmungs-, Deutungs- und Handlungsmustern der Individuen voraus liegen (vgl. z. B. Luckmann 1979: 200). Nur vor dem Hintergrund einer solchen als tertium comparationis fungierenden, universalen Matrix seien die in historischer und kultureller Vielfalt sich darbietenden sozialen Konstruktionen sinnvoll vergleichbar. ${ }^{4}$

Es tut sich nun ein Widerspruch auf zwischen dieser Absicht einer Fixierung ausgewählter soziologischer Kategorien und einer berühmten Passage bei Weber:

»Aber irgendwann wechselt die Farbe: die Bedeutung der unreflektiert verwerteten Gesichtspunkte wird unsicher, der Weg verliert sich in der Dämmerung. Das Licht der großen Kulturprobleme ist weiter gezogen. Dann rüstet sich auch die Wissenschaft, ihren Standort und ihren Begriffsapparat zu wechseln und aus der Höhe des Gedankens auf den Strom des Geschehens zu blicken. Sie zieht jenen Gestirnen nach, welche allein ihrer Arbeit Sinn und Richtung zu weisen vermögen« (Weber 1988: 214).

Nach Weber hat sich die Soziologie dem Wandel ihres Untersuchungsobjekts anzupassen, und zwar sowohl in der Auswahl des für die wissenschaftliche Analyse Relevanten als auch hinsichtlich ihrer Begriffsbildung. Diese Forderung lässt sich auf den ersten Blick schwerlich in Einklang bringen mit dem Unterfangen Schütz', unveränderliche Momente des Sozialen erfassen sowie eine kulturell wie historisch universale Beschreibungssprache für soziale Phänomene zur Verfügung stellen zu wollen. Diese Diskrepanz bildet den Ausgangspunkt der nachstehenden Überlegungen. Die protosoziologische Absicht der Ausformulierung einer universalen Matrix scheint eine gewisse Unverträglichkeit aufzuweisen mit einer Wirklichkeitswissenschaft, die sich an einem im steten Wandel begriffenen Gegenstand orientiert. Will man trotz dieser Feststellung an der Behauptung eines soziologischen Nutzens phänomenologischer Betrachtungen festhalten, so kommt man nicht umhin, die Unverträglichkeit aufzulösen. Zwei Aspekte, auf

3 Die Bedeutung von Husserl für Schütz wird in der Forschung durchaus unterschiedlich bewertet (vgl. jüngst etwa Endreß \& Renn 2004: 36ff oder Barber 2004: 41ff).

4 Luckmann spricht von einer "Metasprache für die je historischen Sprachen, in denen sich die `Daten über menschliches Handeln notwendigerweise darbieten« (Luckmann 1980b: 50).

5 Luckmann hat gelegentlich auf einen Bruch zwischen Schütz und Weber hingewiesen (vgl. etwa Schütz \& Luckmann 1979: 13f), leider jedoch ohne auszuführen, worin dieser seines Erachtens genau besteht (vgl. weiterführend auch Dreher 2007: 14ff). 
die im Folgenden einzugehen sein wird, scheinen mir dabei wesentlich: erstens das methodologische wie methodische Problem des Verhältnisses von empirischer und protosoziologischer Forschung, zweitens - dem zugrunde liegend - die Frage nach dem erkenntnistheoretischen Status der letzteren.

\section{Die reine Soziologie von Ferdinand Tönnies}

Mit Ferdinand Tönnies steht bereits am Beginn der (institutionalisierten) deutschen Soziologie ein Denker, dem an einer Grundlegung der empirischen Sozialforschung gelegen war. Er ist zuvorderst wegen seiner Prägung der Begriffe von Gemeinschaft und Gesellschaft im Gedächtnis der Disziplin verblieben, wobei sein Verständnis derselben einem Wandel unterlag. Hatte er sie laut Untertitel der ersten Auflage seines Hauptwerks im Jahre 1887 noch als »empirische Kulturformen" gedeutet, so verstand er sie von der zweiten Auflage von $1912^{6}$ an als "Grundbegriffe einer reinen Soziologie «" Tönnies stellte damit die Begriffe der Gemeinschaft und Gesellschaft in eine theoriesystematische Ordnung, welche er in der Folgezeit ausführte (vgl. Tönnies 1925, 1965). Er differenziert zwischen allgemeiner und spezieller Soziologie, wobei erstere Sozialbiologie wie -psychologie umfasst. Letztere zerfällt in empirische, angewandte und reine Soziologie. Als empirische Soziologie oder Soziographie begreift Tönnies ungefähr das, was heute als empirische Sozialforschung bezeichnet wird, ${ }^{8}$ und die angewandte Soziologie versucht, den kulturellen Prozess in seinen groben Abläufen zu erklären, ist mithin dynamisch ausgerichtet. Die reine Soziologie schließlich liefert ein feststehendes Gebäude von Theorien und Begriffen so genannter »sozialer Wesenheiten" und ist somit statisch ausgerichtet. Zwischen den drei Bereichen der speziellen Soziologie besteht ein Fundierungsverhältnis: Die angewandte Soziologie wertet Begriffe und Theorien der reinen Soziologie für das Verständnis historischer Entwicklungen aus und mündet in die empirische Soziologie. Die Begriffsbildungen der reinen Soziologie bilden die Grundlage von angewandter und empirischer Soziologie. Neben dieser Aufgabenteilung besteht ferner ein Unterschied hinsichtlich der Vorgehensweise: Die empirische Soziologie verfährt induktiv, die angewandte deduktiv und die reine konstruktiv (1925: 4). So besteht das Geschäft der letzteren im Wesentlichen in der Konstruktion von Begriffen, anhand derer die soziale Wirklichkeit heuristisch erfasst werden kann.

Ideengeschichtlich knüpft die Rede von einer reinen Soziologie an das von Kant in der Kritik der reinen Vernunft eingeführte Konzept einer reinen Naturwissenschaft an, d. h. einer solchen, die eine theoretische Erkenntnis a priori von ihren Gegenständen hat. Illustrieren lässt sich die Funktion der reinen Soziologie anhand der Grundkategorien von Gemeinschaft und Gesellschaft. Diese nennt Tönnies "Normaltypen«, welche die Wirklichkeit nicht klassifizieren sollen, sondern vielmehr Normen zum Ausdruck bringen, welche die empirischen Phänomene mit einem gemeinsamen Bezugspunkt versehen und dadurch erst vergleichbar machen. So schließen sich Gemeinschaft und Gesellschaft im Sinne eines Gegensatzpaars auf der rein begrifflichen Ebene wechselseitig aus, liegen jedoch in der sozialen Realität stets in variierenden Mischungsverhältnissen vor.' Insofern handelt es sich um wissenschaftliche Konstrukte, denn die reine Soziologie "hat es mit Wesenheiten zu tun, die nicht wahrnehmbar sind, sondern als etwas gedacht werden, was zunächst nur im Bewußtsein der menschlichen Personen ist, die in

6 Von René König (1955: 351) als die maßgebliche bezeichnet.

7 Neben bzw. nach Tönnies bezeichneten auch Georg Simmel und Leopold von Wiese ihre Ansätze als reine Soziologie(n). Ich konzentriere mich schon aus Platzgründen ausschließlich auf Tönnies, wobei mir speziell eine Gegenüberstellung mit Simmel vielversprechend erschiene (vgl. z. B. Bond 1991).

8 Mit einer allerdings einseitig quantitativen Ausrichtung.

9 Auf die offenkundige Nähe zu Webers Idealtypenlehre wurde von Tönnies selbst verschiedentlich hingewiesen (vgl. auch Ammassari 1988). 
einer solchen Wesenheit sind und sich bewegen« (1965: 315). Im sozialen Kontext betrifft dies nun wesentlich Verhaltenserwartungen, weshalb sich die reine Soziologie mit Werten und Normen bzw. mit den diese verwirklichenden Bezugsgebilden auseinandersetzt.

Die Auffindung der Normaltypen geschieht im ersten Buch von Gemeinschaft und Gesellschaft aus dem "synonymischen Gebrauche in deutscher Sprache« (1979: 3). Der TönniesExperte Rudolf Heberle $^{10}$ (1959: 395) sieht eine "sprachpsychologische Erkundung, die dann zu der eigentlichen Begriffsbildung hinleitet«. Tönnies selbst (1979: 3) will »den Gegensatz als einen gegebenen darstellen ". ${ }^{11}$ Dessen Begründung indes findet erst im zweiten Buch des Werkes statt, das von "Wesenwille und Kürwille« handelt. ${ }^{12}$ Im Gegensatz zu Webers Idealtypen, welche sich der "gedankliche[n] Steigerung bestimmter Elemente der Wirklichkeit" (Weber 1988: 190) verdanken und als Abstraktionen gerade keine unmittelbare Konvergenz mit der Realität suchen, beinhaltet Tönnies' reine Soziologie eine Lehre der "sozialen Wesenheiten" (Tönnies 1925: 4), die zwar keine materiellen Gegebenheiten darstellen, als im »Medium des gemeinsamen Denkens« (ebd.) verortete Willensakte gleichwohl als real existent begriffen werden: Der »soziologische Sinn [...] hat Gegenbild und Analogie in der Theorie des individualen Willens« (Tönnies 1979: 6). ${ }^{13}$ Der (soziologische) Dualismus von Gemeinschaft und Gesellschaft liegt derart im (psychologischen) Dualismus des menschlichen Willens begründet; Kürwille erzeugt Vergesellschaftung, Wesenwille Vergemeinschaftung. An der Basis von Tönnies' Begriffsbildung stehen letztlich anthropologische Gedanken: "Demnach wird hier von der Essentia des Menschen, nicht von einer Abstraktion, sondern von dem konkreten Inbegriff der gesamten Menschheit, als dem Allgemeinst-Wirklichen dieser Art, ausgegangen« (ebd.: 149).

So erkennt z. B. Plessner (1955: 342) in Tönnies' reiner Soziologie einen "anthropologische[n] Versuch«. König spricht hinsichtlich Tönnies' Willenslehre von einer "Gesamtontologie« (1955: 376) ${ }^{14}$, welche »unaufhaltbar aus der Soziologie herausführt« (ebd.: 382). Statt dessen folge dieser einer "dogmatischen Psychologie, die ganz ungewöhnlich entwicklungsunfähig ist « (ebd.: 355, Hervorh. A. G.); der Mangel an empirischen Quellen entrücke den gesamten Ansatz der Wirklichkeit. Diese Kritik ist im vorliegenden Kontext von herausgehobener Bedeutung, betrifft sie doch unmittelbar das oben angesprochene Verhältnis von prototheoretischer und empirischer Forschung. Tatsächlich behielt Tönnies die Grundkonzeption seines Hauptwerks über acht Auflagen und beinahe ein halbes Jahrhundert hinweg beinahe unverändert bei und wies bspw. die von Herman Schmalenbach in den 1920er Jahren versuchte Erweiterung der Dichotomie von Gemeinschaft und Gesellschaft um einen neuen Typus des Bundes kategorisch zurück (vgl. Schmalenbach 1922, Tönnies 1979: XLIIf). Diese Ablehnung war nur konsequent, denn Tönnies' Begriffssystem ist seiner inhärenten Logik nach allenfalls ausdifferenzierbar, nicht jedoch grundsätzlich erweiterbar (vgl. Bond 1991: 348f). Eine angemessene Reflexion über eine empirisch fundierte Erweiterung der Theorie fehlt in seinem Werk: „Über Empirische Soziologie oder Soziographie hat Tönnies sich in zahlreichen Aufsätzen geäußert, ohne jedoch die Beziehung zur Reinen Soziologie und zur Angewandten Soziologie klarzustellen« (Heberle 1965: XIII).

10 Zugleich dessen Schwiegersohn.

11 König (1955: 388ff) kritisiert die etymologischen Analysen Tönnies' in scharfer Form.

12 So bemerkt denn Tönnies in seiner Vorrede zur ersten Auflage von Gemeinschaft und Gesellschaft folgerichtig, dass das Zweite Buch »in systematischem Gange seine richtigere Stelle vor dem Ersten haben würde« (1979: XXIV; Hervorh. i. O.).

13 Bezeichnenderweise lautet der erste Satz von Gemeinschaft und Gesellschaft. "Die menschlichen Willen stehen in vielfachen Beziehungen zueinander" (Tönnies 1979: 3).

14 Osterkamp (2005: 416-434) unterzieht den Ontologievorwurf von König - neben anderen Vorwürfen desselben - einer Antikritik, belässt es aber bedauerlicherweise bei der wenig erhellenden Feststellung, "um die [...] Bestimmung von Gegenstandsfeldern, Gegenstandsarten und Zugangsbedingungen kommt keine methodisch reflektierte Wissenschaft herum« (ebd.: 418). 
Für das aus heutiger Sicht zu konstatierende Scheitern von Tönnies' reiner Soziologie sind sicherlich nicht zuletzt theorieexterne Faktoren verantwortlich (vgl. König 1955: 350), doch kann dies nicht über die theorieimmanenten Schwächen hinwegtäuschen. So greift der auf wechselseitig bejahende Willensbeziehungen fixierte Theorieansatz für die Erfassung der empirischen Vielfalt sozialer Relationen schlicht zu kurz (vgl. Bond 1991: 347, König 1955: 362ff), zugleich macht es die spezifische Theoriearchitektur praktisch unmöglich, diesen Mangel zu beheben, werden die Grundbegriffe der reinen Soziologie doch als über-empirisch aufgefasst und derart jenseits der Falsifikationsmöglichkeiten empirischer Beobachtung verortet. Die reine Soziologie von Ferdinand Tönnies steht daher als warnendes Beispiel dafür, dass ein dogmatisierendes Verständnis der eigenen Grundannahmen eine Theorie in eine wissenschaftliche Sackgasse zu führen vermag. ${ }^{15}$

\section{Reine Soziologie und Protosoziologie}

Tönnies' Entwurf einer reinen Soziologie weist mehrere Parallelen auf zum von Schütz verfolgten Programm einer phänomenologischen Begründung der Sozialwissenschaften. Gemeingut ist zunächst die Idee, dass es innerhalb der Sozialforschung im umfassenden Sinne einen ausgewiesenen Aufgabenbereich gibt, dessen Gegenstände in irgendeiner Weise "jenseits" der empirischen Beobachtung liegen und dessen Aussagen universale Geltung beanspruchen können: die Theorie der sozialen Wesenheiten bei Tönnies, die Lebenswelttheorie bei Schütz. Diese nehmen je die Schlüsselstellung innerhalb der unterschiedlichen Theoriegebäude ein, sie bilden deren tragende Fundamente. Speziell das Verhältnis zur empirischen Soziologie wird analog aufgefasst; deren Vergleiche sollen durch einen gemeinsamen, statischen Begriffsrahmen erst ermöglicht werden, bei Tönnies mittels der Etablierung feststehender Normaltypen, bei Schütz mittels einer Beschreibung der universellen Strukturen der Lebenswelt. Erreicht wird diese allgemeinste Aussageebene in beiden Fällen mittels des Rückgangs auf Bewusstseinsleistungen des Subjekts, die das Soziale konstituieren. Tönnies bedient sich hierbei historischer Willenstheorien (u. a. bei Schopenhauer), Schütz greift auf die Bewusstseinsanalysen Husserls zurück. Neben allen Differenzen, die ansonsten bestehen, scheinen mir diese Aspekte eine erstaunliche Nähe der beiden Ansätze aufzuzeigen, auf die bereits von König hingewiesen wur$\operatorname{de}^{16}$ (1955: 361). Angesichts dessen liegt die Vermutung nahe, dass die beiden Ansätze auch ähnliche Gefahren bergen.

\section{Protosoziologie als sociologia perennis ${ }^{17}$ ?}

Die Protosoziologie wählt für die Erstellung eines übergeordneten Bezugsrahmens für die empirische Forschung den Anschluss an die Theorie Husserls, eine von allen empirischen Gehalten "gereinigte", transzendentale Psychologie, die auf die "allgemeine Funktionsweise des Apparates `Geist« (Eckart 1981: 25) zielt. Ihre Plausibilität ergibt sich nicht induktiv aus einer möglichst großen Zahl bestätigender Fälle, sondern reflexiv aus der »unmittelbaren Evidenz subjektiver Erfahrung" (Luckmann 1983: 513). Das phänomenologisch Erkannte besitzt Gültigkeit, weil es auf im subjektiven Bewusstsein liegende Bedingungen der Möglichkeit von Wirklich-

15 Nota bene: Die hier formulierte Kritik an Tönnies bezieht sich auf einen spezifischen Aspekt seiner Theorie und will seine Verdienste für die Disziplin - welche das rein Theoretische zudem übersteigen - in keinster Weise in Abrede stellen (für eine komprimierte Würdigung dieser Verdienste vgl. Osterkamp 2005: XIII).

16 Ohne allerdings Schütz namentlich zu erwähnen.

17 Der Begriff spielt an auf das Konzept einer philosophia perennis (immerwährende Philosophie). 
keitssetzungen überhaupt verweist. Erreicht werden soll diese Aussageebene höchster Allgemeinheit mittels der Methoden der epoché und der eidetischen Variation (vgl. z. B. Husserl 1976: $\$ \$$ 31-32, 1999: $\$ \$ 86 f f)$, durch welche die phänomenologische Betrachtung das Wesentliche vom bloß Zufälligen, Nichteidetischen zu trennen sucht. ${ }^{18}$ Dabei macht die epoché, i. e. die schrittweise Einklammerung der in der "natürlichen Einstellung" vollzogenen ontischen Setzungen, den Blick frei für die reinen Bewusstseinsakte und die Ideation deckt auf, welche Elemente dieser Akte für das je in Frage stehende Phänomen konstitutiv sind.

Der Universalitätsanspruch phänomenologischer Aussagen ist indes nicht unumstritten. Schon früh im 20. Jahrhundert hat Johannes Volkelt der Methode der eidetischen Wesensschau ein Überspannen der "singulär-fundierten Wesenserkenntnis" (1918: 449) vorgeworfen. Die Phänomenologie habe zwar Recht mit der Annahme, dass es "Bewusstseinsgebilde« gebe, deren wesenhafte Eigenschaften anhand der Betrachtung eines einzigen Einzelfalles erschlossen werden könnten, sie setze sich jedoch ins Unrecht mit der Ansicht, das Wesen des untersuchten Phänomens brächte sich in intuitiver Anschauung "zur Selbstgegebenheit«. Derart entstehe eine falsche Auffassung vom Gewissheitsstatus phänomenologisch begründeter Sätze. So sage die eidetische Wesenserkenntnis nichts darüber aus, »ob und wann und wo die in dem einen Fall festgestellte Wesenheit sonst noch zu finden ist « (437). Dies gelte sowohl zeitlich im Hinblick auf eigene zukünftige Wahrnehmungen, als auch sozial im Hinblick auf die Wahrnehmungen anderer Personen. Berechtigt sei die Unterstellung einer allgemein gültigen Gewissheit nur dann, wenn sie sich auf solche Bewusstseinsgebilde beziehe, welche eine restlose Durchschaubarkeit auszeichne, wohingegen im Falle von Bewusstseinsgebilden, die »dem Versuche des Durchschauens mehr oder weniger Hemmnisse entgegenstellen « (445), diese Annahme unhaltbar sei. So konstatiert Volkelt:

"Nun ist aber klar: der Selbstgewißheit des Bewußtseins steht der Begriff des Wesens überhaupt nicht zur Verfügung. Von sich aus könnte die reine Gegebenheitsgewißheit nicht zur Unterscheidung des Wesenhaften und Individuell-Eigentümlichen, des Wesenhaften und Erscheinenden gelangen. [...] Auf Grund der bloßen Selbstgewißheit des Bewußtseins ist es ebensowohl möglich, die Aufmerksamkeit auf das zu lenken, was unter begrifflichem Gesichtspunkt als das Individuell-Bestimmte zu beurteilen ist, wie auf das, was unter Anleitung des begrifflichen Denkens sich als das Wesenhafte zu erkennen gibt. Die Selbstgewißheit des Bewußtseins bedarf sonach der methodischen Leitung durch einen dem Denken entstammenden begrifflichen Unterschied. Ohne diese methodische Leitung [...] bestünde nicht die mindeste Sicherheit dafür, daß das Gattungsmäßige, Allgemeine, Wesenhafte an dem Inhalt des [...] Erlebnisses, und nicht vielmehr die gleichgültige individuelle Bestimmtheit in den Umkreis der Selbstgewißheit des Bewußtseins falle» (440f).

Leider wird nun bei Volkelt die Rede von der "restlose[n] Durchschaubarkeit« selbst nicht restlos durchschaubar; er spricht andernorts auch von "Denknotwendigkeiten«. So hat Volkelt das von ihm diagnostizierte Problem zwar nicht gelöst, immerhin jedoch ermöglicht seine Forderung nach "methodischer Leitung" eine Verschiebung des Problems auf die Ebene wissenschaftlicher Praxis. Dort stellt sich dem die phänomenologische Reduktion vollziehenden Wissenschaftler die Frage, von welchen Momenten des empirischen Phänomens er gedanklich abstrahieren darf und von welchen nicht. Verbleibt er bei der Beantwortung dieser Frage ausschließlich im Bereich introspektiver, auf Einzelbetrachtung gründender Bewusstseinsanalyse, so droht ein circulus vitiosus, ein "Zirkel epistemologischer Reflexivität" (Eberle 1993: 305). Vom Nichteidetischen kann der Forscher nur unter der Prämisse abstrahieren, dass er es als solches erkennt. Doch wie kann er das im Zuge reiner Introspektion, wenn er nicht von vornherein über eine Idee des Wesentlichen schon verfügt?

18 Kant spricht in ähnlicher Absicht von "Absonderung« (1993: B 1). 
Lassen Sie mich diesen Punkt - in etwas ungewöhnlicher Form - anhand folgenden, der USamerikanischen TV-Sitcom »Tool Time« entnommenen Dialogs veranschaulichen:

Nachbar 1: Tag Wilson, was machen Sie?

Nachbar 2: $\quad$ Ich schnitz' mir ein Kanu.

Nachbar 1: Schwere Arbeit, hm?

Nachbar 2: Nein, eigentlich nicht Tim. Man nimmt nur 'nen dicken Stamm und schnitzt alles weg, was nicht zu einem Kanu gehört.

Die Pointe dieses Witzes ${ }^{19}$ liegt in einer ironischen Brechung begründet. Selbstverständlich ist das, was in Wilsons Worten so einfach klingt - alles wegzuschnitzen, was nicht zu einem Kanu gehört -, überhaupt nicht einfach, vielmehr handelt es sich um einen höchst anspruchsvollen Akt. Um ihn vollziehen zu können, ist sowohl eine vorgängige und einigermaßen konzise Vorstellung davon, wie das spätere Kanu aussehen soll, unverzichtbar als auch einige Übung in der Durchführung der notwendigen Arbeitsschritte. Auch die phänomenologische Methode der epoché scheint mir nicht bloß theoretische, sondern zudem praktische Vertrautheit mit dem Vorgehen vorauszusetzen. ${ }^{20}$ Im Gegensatz zum Kanuschnitzer allerdings weiß der Phänomenologe im Vorfeld seiner Reduktionen nicht, was deren Ergebnis sein wird, wenn er auch vermutlich eine zumindest vage Vorstellung stets schon mitbringen dürfte. Er will sich im Zuge seiner Arbeit belehren lassen, was die Frage aufwirft, welche Instanz diese Belehrung vollbringen soll. Husserl scheint diese Leistung der Einbildungskraft zuzutrauen, welche im Zuge der eidetischen Variation den gesamten Möglichkeitsspielraum eines Phänomens durchspielt und dabei das in allen Variationen Gleichbleibende und somit Notwendige erkennt. Nun gilt aber die phänomenologische Grundeinsicht in die Perspektivität des menschlichen Weltbezugs auch für den Phänomenologen selbst. Luckmann spricht im Hinblick auf die empirische Forschung "von der perspektivischen Verhaftung des Vergleichers" (2001: 323) und es ist nur konsequent, diese Erkenntnis auch auf den Protosoziologen anzuwenden. Das phänomenologische Verfahren wird von Menschen durchgeführt und ist daher in seinem konkreten Vollzug bedingt durch vorgängige empirische Erfahrungen des variierenden bzw. einklammernden Subjekts, wie sie in biographisch und soziokulturell geformten Deutungsmustern abgelagert sind. Es erscheint daher fraglich, ob die eidetische Variation indifferent gegenüber dem sie vollziehenden Subjekt identische - und damit universal gültige - Ergebnisse zu produzieren vermag. ${ }^{21}$

Die wesentliche Gefahr der rein introspektiv verfahrenden phänomenologischen Betrachtung besteht m. a. W. darin, dass der Phänomenologe dasjenige zum universalen Moment erhöht, was er vor dem Hintergrund des ihm zur Verfügung stehenden Erfahrungshorizontes bloß für notwendig hält, weil ihm keine anders lautenden Erfahrungen zur Verfügung stehen. Wird diese Gefahr nicht reflektiert, so droht die Protosoziologie ihr selbst gestecktes Ziel einer universalen Matrix zu verfehlen. Thomas Eberle spricht von dem "methodologische[n] Problem, wie denn in der egologischen Perspektive die Grenzlinie zwischen universalen lebensweltlichen Invarianten der conditio humana und historisch beziehungsweise kulturspezifischen Variablen gezogen werden kann« (Eberle 1993: 299, vgl. auch 305). Auch Luckmann (1993: 324) weist

19 Der eine Variation eines berühmten Ausspruches von Michelangelo darstellt, wie er in diversen Fassungen überliefert ist und der besagt, dass das Geheimnis der Bildhauerkunst darin bestehe, das Unwesentliche bzw. Überflüssige am Stein wegzunehmen.

20 Kant (1993: B 1) verweist auf die Notwendigkeit »lange[r] Übung« für das Erreichen transzendentaler Erkenntnis. Der geübte Naturforscher wäre in diesem Sinne der geeignetere Transzendentalphilosoph und der geübte Sozialforscher der geeignetere Protosoziologe - ceteris paribus, versteht sich.

21 In der Konsequenz wird damit die strikte Trennung Husserls zwischen eidetischer und empirischer Variation (vgl. z. B. Husserl 1974: 254ff) problematisch. 
auf dieses Problem hin, wenn er - gegen die so genannte "Bilderbuchphänomenologie« gewandt - formuliert: "Es reicht nicht, [...] irgendwelchen anscheinend elementaren Introspektionen universellen Status zuzusprechen«. Der Phänomenologe selbst wird damit gleichsam zum "Einfallstor" der Empirie in die philosophische Deskription - ein Umstand, welcher gleichermaßen das Problem der ethnozentristischen Verzerrung des Eidetischen bedingt wie er auch dessen Lösung beinhaltet, die gerade nicht in der "Selbst-Ausschaltung des Phänomenologen" (Husserl 1976: 137) bestehen kann.

Will man der Gefahr der Konfusion kultureller Deutungsmuster mit invarianten Strukturen der subjektiven Weltorientierung vorbeugen, so kann das nur gelingen - so die im Folgenden zu erläuternde These - durch eine angemessene Verbindung von empirischer Sozialforschung und Protosoziologie. Es gilt, neben der wissenschaftstheoretischen Grenzziehung zwischen Protosoziologie und empirischer Soziologie gleichzeitig den forschungspraktischen Austausch zwischen beiden stark zu machen, d. h. deren Grenze in beide Richtungen als permeabel zu interpretieren, ohne sie dabei zu verwischen. So verstanden liefert die protosoziologische Reflexion zwar die Grundlage empirischer Begriffsbildung, gleichzeitig aber muss die philosophische Analyse ihre Erkenntnisse stets abgleichen mit den empirischen der Soziologie.

Luckmann hat an diesem Punkt das bei Musil entlehnte Bild einer "Parallelaktion" eingeführt (Luckmann 1980c: 100, 1999: 21). Er benennt damit zwei prinzipiell unterschiedliche Verfahren der Beschreibung sowie Erklärung menschlicher Wirklichkeit (philosophisch und soziologisch), die indes zur wechselseitigen Deckung gebracht werden sowie einander ergänzen könnten. Ich will nun die Metapher nicht überstrapazieren, vielleicht aber doch etwas mehr dehnen, als Luckmann dies tut. Nach der mathematischen Definition verlaufen zwei Geraden zueinander parallel, wenn sie in einer Ebene liegen und einander nicht schneiden bzw. im Unendlichen treffen. Somit wäre der Schnittpunkt der beiden aus der empirischen Welt ausgelagert und in eben diesem Punkt scheint mir die Metapher einer (Selbst-)Täuschung Vorschub zu leisten. Richtet man nämlich den Blick nicht auf den Ziel-, sondern den Ausgangspunkt der Bemühungen, so ist klar, dass sich die Geraden in der diesseitigen Welt durchaus schneiden, und zwar in der dem jeweiligen sozio-historischen Apriori verhafteten Perspektive des Forschers, der die Parallelaktion durchführt. Das hat Folgen für die epistemologische Einschätzung eines solchen Unterfangens (vgl. V).

Ich will das Gesagte anhand eines Beispiels verdeutlichen: In seiner V. Cartesianischen Meditation versucht Husserl (1987: 91ff), den phänomenologischen Sinn des Bewusstseinsinhalts "fremdes Ich« zu klären. Dabei klammert er in einem ersten Reduktionsschritt das Fremde, Nicht-Eigene vom Eigenen aus, wobei sich im Wesentlichen der eigene Leib als dasjenige herausstellt, was diese Grenzziehung markiert. Nun hat die Ethnographie, also eine empirische Disziplin, Phänomene wie Ekstase oder Metempsychose ins soziologische Bewusstsein gebracht, bei denen eine differierende Grenzziehung zwischen Eigen- und Fremderfahrung vorzuliegen scheint (vgl. etwa Eliade 1957). Ohne die Gültigkeit solcher Untersuchungen beurteilen zu müssen, lässt sich doch Folgendes festhalten: Sollte sich herausstellen, dass derartige Phänomene nicht als bloße Pathologien zu marginalisieren sind, sondern vielmehr auf eigenständige kulturelle Deutungsmuster verweisen, so müsste dies Auswirkungen auf die transzendental-phänomenologische Analyse der Fremderfahrung haben. Die Grenze zwischen dem Selbst und dem Anderen, wie sie vom westlich sozialisierten, sich in äußerster Bewusstseinsspannung befindlichen Individuum gezogen wird, könnte dann nicht als invariante Bewusstseinsstruktur gelten. Ob solche, der "relativ natürlichen Weltanschauung" des Sozialforschers widersprechenden Kulturmuster existieren, kann ausschließlich die empirische Forschung entscheiden, und so ist denn in diesem Sinne die Genese protosoziologischer Erkenntnis nicht unabhängig von der empirischen. 


\section{Jenseits von Kant?}

Die eben ausgeführten methodologischen Erwägungen haben Auswirkungen auf das epistemologische Verständnis der Protosoziologie. Phänomenologische Analyse vermag Sätze universaler Gültigkeit nur unter der Voraussetzung der "Einheit der Erfahrung bei Menschen " (Luckmann 1980b: 54) zu produzieren. Sie setzt voraus, dass das Bewusstsein des Phänomenologen auf die gleiche Weise "arbeitet" wie dasjenige aller Menschen, sie fußt mithin auf der Unterstellung einer spezifischen Form von Perspektivenreziprozität. Indes liefert die Generalthesis der Reziprozität der Perspektiven keine Begründung menschlicher Gleichheit, sie expliziert lediglich deren allgegenwärtige Unterstellung. Diese Unterstellung ist laut Luckmann eine Prämisse, die sämtlichen kosmologischen Theorien eignet, die aber »im Zuge der Reflexion problematisch werden " (ebd.) kann. Und doch kann das erkenntnisleitende Ideal der Einheit der menschlichen Erfahrung nicht aufgegeben werden, so man eine universale Metasprache soziologischer Beschreibung entwickeln möchte. So zeichnet sich der protosoziologische Ansatz durch eine gewisse innere Gespanntheit aus, insofern die Idee der Einheit menschlicher Erfahrung, welche die empirische Forschung anleiten soll, nicht selbst der Empirie entstammt und induktiv nicht beweisbar ist.

Mit Kant ließe sich an dieser Stelle von einer regulativen Vernunftidee sprechen, wie sie immanent, nicht transzendent zu gebrauchen ist (vgl. Kant 1993: B 670ff). Um aus der Gespanntheit keinen Widerspruch erwachsen zu lassen, wird es m. E. von Vorteil sein, den epistemologischen Status protosoziologischer Aussagen zu überdenken. Ich kann an dieser Stelle nur eine Andeutung geben, in welche Richtung diesbezügliche Überlegungen gehen könnten. In den philosophischen Theorien Philip Kitchers und Saul Kripkes (vgl. z. B. Kitcher 1987, Kripke 1987) findet sich eine Auftrennung der in der früheren Epistemologie als synonym verwendeten Wissensprädikate "apriorisch" und "notwendig". Apriorische Urteile sind für Kitcher und Kripke nicht zwangsläufig - wie noch für Kant - zugleich notwendige Urteile und umgekehrt. ${ }^{22}$ Ermöglicht wird diese Sicht durch einen Ansatz, der das Erkenntnissubjekt in seine Überlegungen mit einbezieht. Ein derartiger Ansatz scheint mir anschlussfähig zu sein für ein solches Verständnis von Protosoziologie, das die Perspektivität des phänomenologischen Forschers berücksichtigt und infolgedessen das, was im Zuge der Introspektion als apriorisch erkannt wird, nicht zugleich als allgemein notwendig setzt. So bleibt die Idee der Einheit der menschlichen Erfahrung und damit invarianter Strukturen derselben zwar weiter gesetzt, fraglich bleibt jedoch stets, ob sie im je konkreten, historisch und biographisch situierten Entwurf auch erreicht wurde. Streicht man dieses Bewusstsein der prinzipiellen Fallibilität selbst protosoziologischer Aussagen, so wird auch der Wert einer phänomenologischen Begründung der Sozialwissenschaften fragwürdig, der nur dann dauerhaft proklamiert werden kann, wenn sie sich offen zeigt gegenüber dem empirischen Erkenntnisfortschritt.

Die vorstehenden Überlegungen argumentierten gegen ein Theorieverständnis, welches das Verhältnis von Protosoziologie und empirischer Sozialforschung als einseitiges Begründungsverhältnis betrachtet, und für ein solches, welches ein Verhältnis der wechselseitigen »Befruchtung " annimmt: Demnach vermag die protosoziologische Reflexion allgemeine Momente des jeweiligen Forschungsfeldes aufzudecken und dieses damit zu strukturieren, doch bleiben ihre Universalaussagen letztlich abhängig von der empirischen Bewährung in der induktiv verfahrenden Forschung. Trennt man diese Verbindung auf, so läuft man Gefahr, Theorieartefakte zu produzieren, deren Nützlichkeit für eine adäquate Beschreibung sozialer Realität in Frage gestellt werden muss. Das Schicksal der reinen Soziologie Tönnies' gibt hierfür ein prominentes Beispiel. Hingegen schwindet die eingangs konstatierte Diskrepanz zu Webers Konzeption einer Wirklichkeitswissenschaft, wenn man die Protosoziologie, in Übereinstimmung mit der

22 Tatsächlich definiert Kripke "notwendig" als metaphysischen, nicht epistemologischen Begriff (für diesen Hinweis danke ich Wolfgang Freitag). 
falsifikationistischen Grundhaltung Poppers (1994), »bescheidener« als »vorläufigen philosophisch begründeten Rückhalt« (Luckmann 1980b: 53) statt als eine sociologia perennis versteht.

\section{Literatur}

Ammassari, Paolo (1988), Tönnies und die typologische Tradition, Annali di Sociologia 4: 1, S. 308-319

Barber, Michael D. (2004), The Participating Citizen. A Biography of Alfred Schutz, Albany: State University of New York Press

Bäumer, Angelica \& Michael Benedikt (Hg. 1993), Gelehrtenrepublik - Lebenswelt. Edmund Husserl und Alfred Schütz in der Krisis der phänomenologischen Bewegung, Wien: Passagen Verlag

Bond, Niall (1991), Noten zu Tönnies und Simmel, in: L. Clausen \& C. Schlüter (Hg.), Hundert Jahre "Gemeinschaft und Gesellschaft". Ferdinand Tönnies in der internationalen Diskussion, Opladen: Leske + Budrich, S. 337-356

Dreher, Jochen (2007), Einleitung, in: Luckmann 2007, S. 7-23

Eberle, Thomas S. (1993), Schütz' Lebensweltanalyse: Soziologie oder Protosoziologie?, in: A. Bäumer \& M. Benedikt (Hg.), S. 293-320

Eckardt, Dietrich (1981), Konstitutionsanalyse. Eine Einführung in die phänomenologische Methode, Bonn: Bouvier

Eliade, Mircea (1957), Schamanismus und archaische Ekstasetechnik, Zürich und Stuttgart: Rascher

Endreß, Martin (2004), Entgrenzung des Menschlichen. Zur Transformation der Strukturen menschlichen Weltbezugs durch Gewalt, in: W. Heitmeyer \& H.-G. Soeffner (Hg.), Gewalt: Entwicklungen, Strukturen, Analyseprobleme, Frankfurt a.M.: Suhrkamp, S. 174-201

Endreß, Martin \& Joachim Renn (2004), Einleitung der Herausgeber, in: Schütz 2004, S. 8-66

Heberle, Rudolf (1959), Tönnies, Ferdinand, in: Handwörterbuch der Sozialwissenschaften, Bd. 10, Tübingen: Mohr, S. 394-397

Heberle, Rudolf (1965), Geleitwort zur Neuausgabe, in: Tönnies 1965, S. XI-XIX

Husserl, Edmund (1974), Formale und transzendentale Logik. Versuch einer Kritik der logischen Vernunft (Husserliana Bd. XVII), hg. v. Paul Janssen, Den Haag: Nijhoff

Husserl, Edmund (1976), Ideen zu einer reinen Phänomenologie und phänomenologischen Philosophie. Erstes Buch: Allgemeine Einführung in die reine Phänomenologie (Husserliana Bd. III/1), Den Haag: Nijhoff

Husserl, Edmund (1987), Cartesianische Meditationen. Eine Einleitung in die Phänomenologie, Hamburg: Meiner

Husserl, Edmund (1999), Erfahrung und Urteil. Untersuchungen zur Genealogie der Logik, Hamburg: Meiner

Kant, Immanuel (1993), Kritik der reinen Vernunft, Hamburg: Meiner

Kitcher, Philip (1987), Apriority and Necessity, in: P. Moser (Hg.), S. 190-207

König, René (1955), Die Begriffe Gemeinschaft und Gesellschaft bei Ferdinand Tönnies, KZfSS 7, S. $348-420$

Kripke, Saul A. (1987), A priori Knowledge, Necessity, and Contingency, in: Moser (Hg.), S. 145-160

Luckmann, Thomas (1979), Phänomenologie und Soziologie, in: W. Sprondel \& R. Grathoff (Hg.), Alfred Schütz und die Theorie des Alltags in den Sozialwissenschaften, Stuttgart: Enke, S. 196-206

Luckmann, Thomas (1980a), Lebenswelt und Gesellschaft: Grundstrukturen und geschichtliche Wandlungen, Paderborn: Schöningh (UTB)

Luckmann, Thomas (1980b), Philosophie, Sozialwissenschaft und Alltagsleben, in ders. 1980a, S. 9-55 (neu in ders. 2007, S. 25-61)

Luckmann, Thomas (1980c), Aspekte einer Theorie der Sozialkommunikation, in ders. 1980a, S. 93-121 (neu in ders. 2007, S. 91-111)

Luckmann, Thomas (1983), Eine phänomenologische Begründung der Sozialwissenschaften?, in: D. Henrich (Hg.), Kant oder Hegel? Über Formen der Begründung in der Philosophie, Stuttgart: KlettCotta, S. 506-518

Luckmann, Thomas (1993), Schützsche Protosoziologie?, in: A. Bäumer \& M. Benedikt (Hg.), S. 321-326

Luckmann, Thomas (1999), Wirklichkeiten: individuelle Konstitution, gesellschaftliche Konstruktion, in: R. Hitzler et al. (Hg.), Hermeneutische Wissenssoziologie, Konstanz: UVK, S. 17-28 (neu in ders. 2007, S. 127-137) 
Luckmann, Thomas (2001), Sinn in Sozialstruktur, in: A. Brosziewski et al. (Hg.), Moderne Zeiten. Reflexionen zur Multioptionsgesellschaft, Konstanz: UVK, S. 311-325 (neu in ders. 2007, S. 138-150)

Luckmann, Thomas (2007), Lebenswelt, Identität und Gesellschaft, hg. von Jochen Dreher, Konstanz: UVK

Moser, Paul K. (Hg. 1987), A priori Knowledge, Oxford: University Press

Osterkamp, Frank (2005), Gemeinschaft und Gesellschaft: Über die Schwierigkeiten einen Unterschied zu machen. Zur Rekonstruktion des primären Theorieentwurfs von Ferdinand Tönnies, Berlin: Duncker \& Humblot

Plessner, Helmuth (1955), Nachwort zu Ferdinand Tönnies, KZfSS 7, S. 341-347

Popper, Karl R. (1994), Logik der Forschung, Tübingen: Mohr

Schmalenbach, Herman (1922), Die soziologische Kategorie des Bundes, Die Dioskuren, 1. Bd., S. 35-105

Schütz, Alfred (1971), Wissenschaftliche Interpretation und Alltagsverständnis menschlichen Handelns, in ders., Gesammelte Aufsätze, Bd. I: Das Problem der sozialen Wirklichkeit, Den Haag: Nijhoff, S. $3-54$

Schütz, Alfred (2004), Der sinnhafte Aufbau der sozialen Welt. Eine Einleitung in die verstehende Soziologie (ASW II), Konstanz: UVK

Schütz, Alfred \& Thomas Luckmann (1979), Strukturen der Lebenswelt, Bd. I, Frankfurt a.M.: Suhrkamp

Schütz, Alfred \& Thomas Luckmann (1984), Strukturen der Lebenswelt, Bd. II, Frankfurt a.M.: Suhrkamp

Sofsky, Wolfgang (1993), Die Ordnung des Terrors: Das Konzentrationslager, Frankfurt a.M.: Fischer

Tönnies, Ferdinand (1925), Einteilung der Soziologie, Zeitschrift für die gesamte Staatswissenschaft, Bd. 79, S. $1-15$

Tönnies, Ferdinand (1965), Einführung in die Soziologie, Stuttgart: Enke

Tönnies, Ferdinand (1979), Gemeinschaft und Gesellschaft: Grundbegriffe der reinen Soziologie, ND d. 8. Aufl. v. 1935, Darmstadt: Wissenschaftliche Buchgesellschaft

Volkelt, Johannes (1918), Gewissheit und Wahrheit: Untersuchung der Geltungsfragen als Grundlegung der Erkenntnistheorie, München: Beck

Weber, Max (1988), Die »Objektivität« sozialwissenschaftlicher und sozialpolitischer Erkenntnis, in ders., Gesammelte Aufsätze zur Wissenschaftslehre, Tübingen: Mohr, S. 146-214 